\title{
Predição de Acidemia Fetal Mediante Dopplervelocimetria do Ducto Venoso em Gestações com Insuficiência Placentária
}

\author{
Prediction of Fetal Acidemia by Doppler Velocimetry of the Ductus Venosus in Gestations \\ with Placental Insufficiency
}

Autor: Francisco Herlânio Costa Carvalho

Orientador: Prof. Dr. Antonio Fernandes Moron

Tese de Doutorado apresentada ao Departamento de Obstetrícia da UNIFESP-Escola Paulista de Medicina, em 24 de maio de 2004.

Objetivos: investigar a possibilidade de predição de acidemia ao nascimento em gestações com insuficiência placentária com o uso da dopplervelocimetria do ducto venoso e dos índices venoso-arteriais, definindo seus pontos de corte e determinando qual o melhor parâmetro nessa predição.

Pacientes e Métodos: trata-se de estudo transversal e prospectivo que analisou 47 gestações únicas após a 26a. semana no Hospital São Paulo (UNIFESP) e na Maternidade-Escola Assis Chateaubriand (UFC). Foram excluídos fetos com anomalias estruturais ou cromossômicas. A dopplervelocimetria foi realizada a menos de 24 horas do parto. Amostra de sangue arterial umbilical foi coletada imediatamente após o nascimento. Construiu-se curva ROC para cada parâmetro da dopplervelocimetria, do DV e dos indices venoso-arteriais. Determinaram-se os pontos de corte e posteriormente foram calculados a sensibilidade, especifici- dade, VPP, VPN e acurácia. Os parâmetros foram comparados entre si através do teste de MacNemar.

Resultados: as velocidades S, D e A do DV não se mostraram boas preditoras de acidemia ao nascimento. $\mathrm{O}$ IPV (área sob a curva ROC 0,79, p = 0,003), as relações S/A e (S-A)/S (área 0,818, p = 0,001) do DV e o índice IP DV/IP ACM (área 0,785, p = 0,004) mostraram ser fortemente relacionados com acidemia fetal. Os pontos de corte calculados foram: IPV = 0,76; S/A = 2,67; $(\mathrm{S}-\mathrm{A}) / \mathrm{S}=$ 0,63 e IP DV/IP ACM = 0,582.

Conclusões: Os índices ângulo-independentes do Doppler do DV e o índice IP DV/IP ACM mostraram-se adequados na predição de acidemia ao nascimento nessa população. Os parâmetros são estatisticamente similares quanto ao poder de predição.

Palavras-chave: Acidemia fetal. Dopplervelocimetria. Ducto venoso. Insuficiência placentária.

\section{Efeito do Implante de Etonogestrel sobre a Agregáação Plaquetária de Mulheres Hígidas}

\section{Effect of Etonogestrel Implant on Platelet Aggregation in Healthy Women}

Autora: Carolina Sales Vieira Macedo

Orientador: Prof. Dr. Marcos Felipe Silva de Sá

Dissertação de Mestrado apresentada à Faculdade de Medicina de Ribeirão Preto - USP, Departamento de Ginecologia e Obstetrícia, em 28 de junho de 2004.

Objetivo: avaliar o efeito do implante subdérmico de etonogestrel sobre a agregação plaquetária de mulheres hígidas, em seis meses de tratamento.

Casuística e Métodos: vinte e quatro mulheres saudáveis e voluntárias foram selecionadas neste estudo longitudinal e prospectivo, para usar um implante contraceptivo subdérmico de etonogestrel (metabólito biologicamente ativo do desogestrel). A agregação plaquetária foi avaliada em todas as mulheres, exceto uma, no período pré-inserção e após um, três e seis meses da inserção do implante. A agregação plaquetária foi induzida com adrenalina 50 $\mu \mathrm{M}$, colágeno $10 \mu \mathrm{g} / \mathrm{ml}$, colágeno $5 \mu \mathrm{g} / \mathrm{ml}$, ADP $35 \mu \mathrm{M}$ e ADP $17,5 \mu \mathrm{M}$. A análise estatística foi feita com o teste de Wilcoxon para comparar a diferença entre cada período de tratamento com os valores pré-tratamento.

Resultados: houve uma redução transitória, estatisticamente significativa, na mediana do percentual má- ximo de agregação plaquetária de 27\%, 14\% e 11\%, respectivamente, com colágeno $5 \mu \mathrm{g} / \mathrm{ml}$, adrenalina $50 \mu \mathrm{M}$ e colágeno $10 \mu \mathrm{g} / \mathrm{ml}$, observada um mês após a inserção do implante comparado ao valor pré-inserção $(\mathrm{p}<$ 0,05). A agregação plaquetária com esses agonistas retornou ao seu valor basal, após seis meses da inserção. Com outros agonistas, como o ADP $35 \mu \mathrm{M}$ e $\mathrm{ADP}$ 17,5 $\mu \mathrm{M}$, não se observou o mesmo fenômeno. Conclusão: os resultados deste estudo mostram, pela primeira vez, que o uso do implante de etonogestrel está associado à redução transitória, mas significativa, da agregação plaquetária, observada em um mês de uso do contraceptivo, a qual retorna a seus valores normais em seis meses da inserção do implante.

Palavras-chave: Contracepção. Agregação plaquetária. Coagulação sangüínea. Progestagênios. 